\title{
The Role of Fitbits in Corporate Wellness Programs: Does Step Count Matter?
}

\author{
Laurie Giddens \\ Baylor University \\ Laurie_Giddens@baylor.edu
}

\author{
Dorothy Leidner \\ Baylor University \\ Lund University \\ Dorothy_Leidner@baylor.edu
}

\author{
Ester Gonzalez \\ California State University, Fullerton \\ esgonzalez@fullerton.edu
}

\begin{abstract}
Striving to promote the health and well-being of their workforce and decrease insurance expenditures, many organizations are incorporating wearable fitness trackers into their corporate wellness programs. Research suggests that these devices encourage individuals to make healthier choices; however, little is known about the drivers and impact of fitness trackers on employee health and wellbeing. We seek to fill this knowledge gap by investigating the following research questions: (1) what is the impact of wearable fitness device use on employee health and well-being? (2) what is the impact of wearable fitness device use on step count? Our results indicate that extended use has a positive impact on employee well-being and step count, which fully mediates the relationship between feature use and health. These findings have implications for research into wearable fitness trackers and, more specifically, for research on the uses of wearable fitness devices in corporate wellness programs.
\end{abstract}

\section{Introduction}

Striving to both promote the health and wellbeing of their workforce and decrease insurance expenditures, many organizations are incorporating wearable fitness trackers into their corporate wellness programs. In order to improve workforce health and well-being, corporations have spent nearly $\$ 100$ billion on workplace wellness programs since the 1970s [41]. Research indicates that well-designed corporate wellness programs can improve job satisfaction, employee mood, and employee fitness levels while also reducing job stress and employee absenteeism [16]. These programs may also improve certain health conditions such as diabetes [11]. However, corporate wellness programs have been historically plagued by a lack of employee participation and difficulty tracking employee progress. With the rapid emergence of wearable technology companies are turning to wearable devices to invigorate their corporate wellness programs [30].

Wearable devices are computers that are embedded into clothing or accessories that provide self-tracking data to the user. Among the most popular of such self-tracking devices for consumers are wearable fitness devices such as Fitbit, Jawbone, and Garmin Forerunner. These devices track the number of steps taken, sleep patterns, heart rate and calories expended through a wristband worn by the user. Already nearly 2,000 companies worldwide offer fitness tracking devices to employees [40] and more than 13 million wearable fitness devices are projected to be in use in corporate wellness programs by 2018 [30]. Companies such as Barclays and Target distribute Fitbits to their employees and offer incentives to participants to use the devices and share their information [34]. Fitness tracking devices offer unique features aimed at engaging individuals in a healthy lifestyle. By using these devices in tandem with corporate wellness programs, organizations hope to increase the effectiveness of their wellness initiatives.

Many corporate wellness programs incorporate wearable fitness devices in order to encourage employees to increase their physical activity, which is generally measured by a daily step count. Studies on health and fitness indicate that individuals who get 10,000 steps a day, which equals a distance of five miles, observe many health benefits [9]. As such, companies utilizing fitness trackers in their wellness programs typically encourage or incentivize participants to walk as many steps a day as possible, with a goal of at least 10,000 steps. Research suggests that wearable fitness devices encourage individuals to be more active and to make healthy choices [15]; however, we do not know what aspects 
of these devices drive activity and what impact fitness trackers have on employee health and wellbeing. We seek to fill this knowledge gap by investigating the following research questions: (1) what is the impact of wearable fitness device use on employee health and well-being? (2) what is the impact of wearable fitness device use on daily step count?

We investigate these research questions at a company using Fitbits as part of its corporate wellness program. Our findings have implications for research into wearable fitness trackers and, more specifically, for research on the uses of wearable fitness devices in corporate wellness programs. This paper is organized as follows. In section two we present a literature review on wearable fitness devices and the impacts on health and well-being. We then explain our research model and hypotheses. Next, we present our data analysis and results. Finally, we discuss our results and the implications for theory and practice.

\section{Background literature}

According to Gartner, there will be 500 million wearable devices in the hands of consumers by 2020 [27]. Many wearable devices are used for the purpose of self-tracking, or self-quantifying, which is the act of collecting data about oneself to change behavior and improve personal outcomes [46]. A Pew Internet study on self-tracking revealed that $60 \%$ of Americans indicate they engage in self-tracking and $21 \%$ of those use technology to do so [19]. Sensors embedded in wearable technologies have made selfquantifying possible, enabling individuals to track everyday events such as mood, health indicators and physical activity. Although wearable devices embody a variety of forms and functions, a majority of devices are used to improve user health [42]. Health care providers now use wearable devices to track health and well-being indicators of patients. For instance, a physician may supply a patient with a device that provides real-time information on patient blood pressure, heart rate, temperature, or blood sugar level. Additionally mobile apps, coupled with wearable devices, are used to encourage wearers to be physically active and make healthy choices [14,23].

Given that wearable technology is touted as a means for individuals to improve their health and well-being, it follows that wearable technology is an ideal tool to use in corporate wellness programs that endeavor to create a happy and healthy workforce. While having healthy employees may alleviate rising insurance premiums, it may also improve productivity and performance in the workplace [47]. In a 2013 study, employees with a healthy diet and in good physical health were associated with better job performance and low absenteeism [32]. Similarly, employee well-being is also posited to improve employee performance. Scholars have found that employee well-being can improve employee performance, job satisfaction, organizational commitment, organizational citizenship behavior and ultimately, reduce employee turnover [2,45]. Employees who report high levels of well-being also report lower levels of work stress and lower rates of absenteeism [2,39]. Given the impact of a happy and healthy workforce on organizations, and on public health, it is important to understand how wearable technology may help organizations achieve the benefits of having healthy employees.

Both health and well-being have become an important variable of interest in information systems research. There is considerable research on the role of information systems in improving healthcare and health outcomes [1]. While studies on health care information systems is thriving, research on IS and well-being is less prevalent. Research on IS and wellbeing investigate the effect of technology on all levels of well-being including personal well-being [6], employee well-being [33], and nation well-being [20]. IT research has also considered the impact of technology use on quality of life [20,28]. However, the research on IT and personal well-being is still lacking. In this paper, we address this gap in research by investigating the effect of Fitbit use on well-being, and ultimately, health. Also, we investigate how Fitbit use might encourage physical activity.

\section{Hypothesis development}

In this section we present a research model with exploratory hypotheses. Our goal is to explore the impact of wearable fitness device use on the users' physical activity level, health and well-being. For this research, the participants are using an employer provided Fitbit as part of a corporate wellness program. Our research model can be seen in Figure 1. 


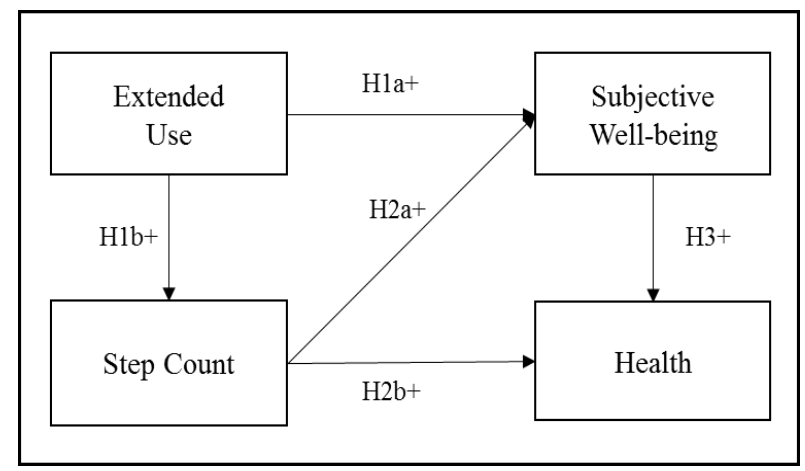

Figure 1. Research model

\subsection{Extended feature use}

While tracking steps is important, wearable fitness devices, such as Fitbit, have many other features available to users through its App, which may help motivate individuals to exercise and make healthier choices. These features go beyond the primary function of Fitbit as a pedometer and may be used for self-tracking other activities and to connect with other users for support and motivation. The Fitbit, which is the wearable fitness device used in this study, has nearly two dozen features available to users. Some of the self-tracking features include sleep monitoring, stair counting, food tracking, workout tracking, goal setting and using the Fitbit App in conjunction with other health and fitness apps. Social features on the App include connecting with other users, starting group competitions, or sending taunts and cheers to other users. We measure Fitbit breadth of use by counting the number of features the user employs in addition to step counting. We refer to this breadth of use as extended use [37]. It is important to measure the extended use of Fitbits because it is the features of wearable fitness trackers that help users change their behavior and create healthy habits [3]. Also, feature use is often overlooked in corporate wellness programs in that employees are encouraged to track their steps, but are not necessarily encouraged to use all the features available to them through fitness device apps. Researchers have found that extended use of technology improves user well-being. For instance, Carter et al. [6] found that extended use of tablets led to higher subjective well-being. There is also anecdotal evidence that wellness programs that incorporate wearable fitness devices have happier employees [30]. As such, we argue that:

Hla: Extended feature use has a positive impact on subjective well-being.
Studies investigating the effect of mobile apps on encouraging physical activity indicate that apps which include behavior change techniques were more effective at boosting fitness activities [12]. Behavior change techniques, or features, include goal setting, social support, user feedback, and activity instruction [12]. These features help to educate and motivate users to improve their fitness levels. A study on the effectiveness of pedometers found that individuals who set a step goal when using a pedometer got a higher number of steps each day than those who did not use a step goal [4]. Additionally, users who connect with at least one other Fitbit user get more steps per day than those who track their steps without the support of other users [17]. Features that help individuals track their progress have also been shown to increase physical activity [35]. It follows that the more Fitbit features users employ to motivate and support their fitness, the higher their daily step count will be. For this reason, we hypothesize:

H1b: Extended feature use will have a positive impact on daily step count.

\subsection{Step count}

It is well-known in the healthcare community that physical activity, including walking, is related to positive health outcomes [25] while sedentary lifestyles are associated with negative health outcomes [43]. Physical activity is said to improve a variety of health outcomes including body mass index, diabetes, and heart disease [25,36]. However, individuals, especially those in the Europe and North America, are increasingly living more sedentary lifestyles than in previous years. Research indicates that higher step counts are associated with health benefits such as lower body mass index [4]. Physical activity is also associated with happiness and wellbeing [18]. In a literature review on the impacts of physical activity on physical and mental health, Penedo and Dahn [36] found that individuals who exercised had higher mental health outcomes, including better mood states, than those with less physical activity levels. Moreover, those who exercised had reduced rates of heart disease [36].

One way to measure physical activity is by counting the number of steps an individual takes during the day. The medical community has established that 10,000 steps per day, around 5 miles of walking, is the standard for daily exercise required to see positive health outcomes [9]. Wearable fitness devices function as pedometers, or step counters, and help individuals track their steps each day. As such, we use step count as a measure of physical activity. 
Given the research on the positive impact of exercise on health and well-being, we assert that:

H2a: Daily step count has a positive impact on subjective well-being.

H2b: Daily step count has a positive impact on health.

\subsection{Subjective well-being and health}

Well-being is an important predictor of physical health. In a recent study, well-being was found to positively mediate the relationship between IT use and health [6]. Medical research also highlights the positive impact of personal well-being and physical health. For example, the health benefits of positive emotions has been shown to improve health outcomes. McCraty et al. [31] investigated the effect of emotions on heart rate variance (HRV) and observed that positive emotions led to changes in HRV which might improve hypertension and temper the possibility of sudden death in patients with heart disease. Well-being was also found to contribute to both health and longevity in healthy populations [13]. Therefore, we posit:

H3: Subjective well-being has a positive impact with health.

\section{Research method}

\subsection{Data collection}

Participants for our study are employees at a financial institution located in the southern United States, which we will refer to as FIT Bank. This company was chosen because it started a wellness program in 2015 that provides each employee with a free Fitbit Flex or a discount on an advanced Fitbit model. Like many companies, FIT Bank has suffered from high health insurance claims and high absenteeism. It was in response to these costs that officials at FIT Bank decided to start a wellness program. Currently, FIT Bank operates 24 banking centers and has approximately 280 employees. The bank distributed Fitbits to participating employees and provides incentives for employees to reach 10,000 steps a day, an amount which the medical field suggests improves health and fitness. FIT Bank hopes that its wellness program will decrease the high insurance costs that have been weighing heavily on the bank every year. It is through this wellness initiative that the bank began to offer individual incentives to participate in the program and later moved into team incentives in order to promote active participation in the health program.

An online survey was used to gather data to test our exploratory research model. We used Qualtrics to administer the survey; no paper surveys were used. Our sample consisted of employees at FIT Bank who are participating in the wearable tech wellness program. The survey was sent out to all participating employees at FIT Bank. A total of 87 employees responded to the survey; however 34 respondents did not complete all the questions. As such, our data collection yielded an $\mathrm{N}=53$. Employees at FIT bank completed the survey on a voluntary basis and were encouraged to participate by the marketing director. A description of our sample can be seen in Table 1.

Table 1. Sample demographics

\begin{tabular}{|l|c|l|c|}
\hline Gender & \multicolumn{2}{|l|}{ Age } \\
\hline Female & $79 \%$ & $18-24$ & $4 \%$ \\
\hline Male & $21 \%$ & $25-34$ & $25 \%$ \\
\hline Marital S tatus & $9 \%$ & $45-54$ & $23 \%$ \\
\hline Single & $79 \%$ & $55-64$ & $34 \%$ \\
\hline Married & $0 \%$ & $65-74$ & $11 \%$ \\
\hline Separated & $9 \%$ & $75-$ Above & $2 \%$ \\
\hline Divorced & $2 \%$ & & $2 \%$ \\
\hline Widowed & $9 \%$ & Asian & \\
\hline Education Level & $38 \%$ & Black & $2 \%$ \\
\hline High School & $21 \%$ & White & $6 \%$ \\
\hline Some College & $23 \%$ & Hispanic & $21 \%$ \\
\hline Associate Degree & $9 \%$ & Native American & $0 \%$ \\
\hline Bachelor's Degree & $0 \%$ & Other & $0 \%$ \\
\hline Master's Degree & & \\
\hline Doctoral Degree & & \\
\hline
\end{tabular}

\subsection{Operationalization of variables}

In order to investigate extended use, we count the number of features that participants indicated they used [6]. Respondents were asked to indicate which Fitbit app features they used. The features listed on the survey were taken from the Fitbit website. Step count is the average daily step count achieved by the user. This number was given by the respondents. In order to capture well-being and health, we used measures from the PERMA profiler instrument [5]. The items in this instrument are based on Seligman's research on well-being which identifies five specific measurable domains that make up subjective wellbeing [38]. The PERMA-profiler assess well-being 
by asking respondents about these specific areas which include: positive emotions, engagement, relationships, sense of meaning, accomplishment. The instrument contains 15 questions for well-being, three for each domain. The items are then combined into one score, or index, which measures a person's overall sense of well-being [5]. The Profiler also includes separate items that capture an individual's sense of physical health. The PERMA-Profiler has been used in a variety of recent studies to measure well-being [6,24,29].

\section{Analysis and results}

We used partial least squares (PLS) to analyze our data. PLS simultaneously estimates the relationship between indicators and latent variables, or measurement paths, and the path relationships in the model, structural paths [7]. The goal of PLS is to maximize the explained variance of the endogenous latent variables and is primarily used for theory development and the prediction of constructs [22]. PLS is robust for non-normal data, and small sample sizes [8,22]. Given that objective of this research exploratory and the size of our sample, we chose PLS to test our research model. The PLS analysis has two stages: (1) the analysis of the measurement (outer) model followed by (2) an assessment of the structural (inner) model [7]. As such, we first examine our measurement model and then move to the structural model.

\subsection{Measurement model}

We first examined the measurement model to assess the reliability and validity of the indicators to the health latent variable. The bootstrap method, which generates 5000 random samples from the original data set $(\mathrm{N}=53)$, was used to produce parameter estimates and standard errors across our total sample (Hair et al. 2013). We evaluated the measurement model by looking at the internal reliability and convergent and discriminant validity of our latent variable [22]. Since our measures for extended use and step count are indices, we will only assess the internal consistency reliability of health. We assessed reliability of our latent variable using Cronbach's alpha, as shown in Table 2. In order to demonstrate the reliability of the latent variables, Cronbach's alpha should be at .7 or above [21] and the score for Health is .93. The Average Variance Extracted (AVE) value for health was .878. An AVE score above .5 indicates that the LV explains over $50 \%$ of the item variance, which demonstrates that
Health has convergent validity. Item loadings for Health ranged from .934 to .942 , which is above the .7 recommendation and indicates that Health has convergent validity [21].

Table 2. Reliability and correlations

\begin{tabular}{|l|c|r|r|r|r|}
\hline & \multirow{2}{*}{\begin{tabular}{r} 
Alpha \\
\cline { 4 - 6 }
\end{tabular}} & 1 & 2 & 3 & 4 \\
\hline 1. Extended Use & n/a & $\mathbf{1 . 0 0}$ & & & \\
\cline { 2 - 6 } & 0.93 & 0.50 & $\mathbf{1 . 0 0}$ & & \\
\hline 2. Health & $\mathrm{n} / \mathrm{a}$ & 0.37 & 0.58 & $\mathbf{1 . 0 0}$ & \\
\hline 3. Step Count & $\mathrm{n} / \mathrm{a}$ & 0.51 & 0.66 & 0.44 & $\mathbf{1 . 0 0}$ \\
\hline 4. Well-being & & & &
\end{tabular}

Since our model did not contain any formative constructs, we proceeded to the evaluation of the structural model.

\subsection{Structural model}

The structural model shows the relationship between each of the latent variables. We evaluate the outer model by examining the structural path coefficients, the R2 values, and effect size. First we assessed the path coefficients and their corresponding t-values to determine if our hypothesis were supported, as shown in Table 3. We found support for all our hypotheses. Extended use explained the highest amount of variance in subjective well-being $(\beta=.400, p<.001)$, followed by step count $(\beta=.293$, $\mathrm{p}<.01)$. The path coefficients for extended use on step count is $(\beta=.368, \mathrm{p}<.01)$. Subjective wellbeing explains the highest amount of variance in health $(\beta=.498, \mathrm{p}<.001)$, followed by step count $(\beta$ $=.357, \mathrm{p}<.01)$. We also examined the magnitude of these effects with Cohen's f2. Table 3 shows that subjective well-being has an effect size of .427 on behavioral intention. According to Cohen [10], this constitutes a medium effect size. The other paths represent a small on their respective endogenous variable. Our model with path coefficients and R2 can found in Figure 2.

Table 3. Model results, $t$ statistics, and effect size

\begin{tabular}{|c|c|c|c|c|}
\hline Constructs & $\begin{array}{l}\text { Path } \\
\text { Coefficients }\end{array}$ & $\begin{array}{l}\mathrm{T} \\
\text { Statistics }\end{array}$ & \begin{tabular}{|l}
$\mathrm{P}$ \\
Values
\end{tabular} & $\begin{array}{l}\text { Effect } \\
\text { Size }\end{array}$ \\
\hline \multicolumn{5}{|l|}{ Step Count, R2 = .136 } \\
\hline Extended Use $>$ StepCount & 0.368 & 2.803 & 0.005 & 0.157 \\
\hline \multicolumn{5}{|l|}{ Psych. Well-being, R2 = .332 } \\
\hline Extended Use > Well-being & 0.400 & 4.145 & 0.000 & 0.207 \\
\hline Step Count $>$ Well-being & 0.293 & 2.872 & 0.004 & 0.111 \\
\hline \multicolumn{5}{|l|}{ Physical Health, $\mathrm{R} 2=.532$} \\
\hline Step Count $>$ Health & 0.357 & 2.791 & 0.005 & 0.220 \\
\hline Well-being $>$ Health & 0.498 & 4.474 & 0.000 & 0.427 \\
\hline
\end{tabular}


The variance explained (R2) for each endogenous variable is shown in Table 3. The R2 for step count is .136 , which indicates that extended use accounts for $13 \%$ of variance in daily step count. The R2 for psychological well-being is .332 demonstrating that $33 \%$ of the variance explained in well-being can be attributed to extended use and step count. Finally, the R2 for physical health is .532 which means that $50 \%$ of the variance in physical health is explained by step count and psychological well-being.

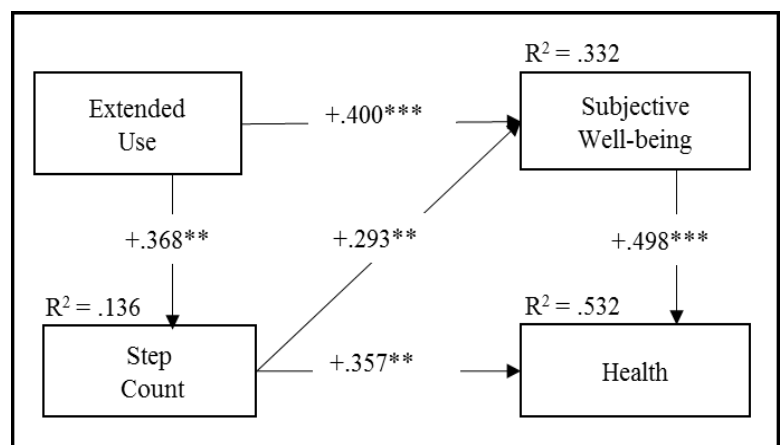

Figure 2. Model results and path coefficients

\subsection{Post-hoc analysis}

We assessed the mediation effect of step count and well-being by evaluating the indirect effect point estimate and confidence intervals. The results can be seen in Table 4. We found that step count fully mediates the relationship between extended feature use and health and partially mediates the relationship between extended feature use and well-being. Additionally, we found that subjective well-being fully mediates the relationship between extended use and health, while it partially mediates the relationship between step count and health. We used confidence intervals to report indirect effects [44]. Our confidence intervals do not contain zero, which indicates that an indirect is present.

Table 4. Indirect effects

\begin{tabular}{|c|c|c|c|c|c|}
\hline \multirow{3}{*}{ Indirect Effect } & & \multirow{2}{*}{\multicolumn{2}{|c|}{$\begin{array}{c}\begin{array}{c}\text { Percentile } \\
\text { bootstrap }\end{array} \\
\text { 95\% confidence } \\
\text { Interval } \\
\end{array}$}} & \multirow{2}{*}{\multicolumn{2}{|c|}{$\begin{array}{c}\text { Bias Corrected } \\
\text { bootstrap } \\
\text { confidence } \\
\text { Interval } \\
\end{array}$}} \\
\hline & \multirow[t]{2}{*}{$\begin{array}{c}\text { Point } \\
\text { Estimate }\end{array}$} & & & & \\
\hline & & Lower & Higher & Lower & Higher \\
\hline Extended Use $>$ Health & 0.384 & 0.220 & 0.576 & 0.215 & 0.574 \\
\hline Extended Use $>$ Well-Being & 0.108 & 0.012 & 0.234 & 0.012 & 0.236 \\
\hline Step Count $>$ Health & 0.146 & 0.033 & 0.256 & 0.031 & 0.257 \\
\hline
\end{tabular}

\section{Discussion and Conclusion}

Our research examines the role of wearable fitness devices in helping organizations foster a happy and healthy workforce. First, we found that extended use of Fitbit features has a positive impact on daily step count. Since corporate wellness programs that use wearable fitness devices often encourage employees to get at least 10,000 steps a day, it is helpful to understand how users can effectively utilize wearable fitness devices to help maximize their step count. These results suggest that employing the features available with the Fitbit app helps to motivate and encourage users to get more steps. As such, companies using wearable fitness devices should not only encourage step count, but design wellness programs that encourage employees to use the breadth of features available. For example, FIT Bank used a group competition approach in which employees form teams and compete against other teams to achieve the highest number of steps. The team with highest number receives a reward. This team set up not only encourages step count, but it also encourages participants to use certain features, other than step tracking. Case in point, the participants at FIT Bank connect with other users on their team, start competitions, and can motivate and encourage one another, all of which is facilitated with the Fitbit app. Ultimately, corporate wellness program managers will benefit by designing programs that encourage employees to engage in both social and tracking features available to them.

Along with reduced health insurance premiums, corporate wellness programs are said to improve employee well-being. The findings of our study suggest that employee well-being can be enriched when users engage in extended use of the Fitbit app, regardless of the amount of steps they take per day. One explanation as to why the very wearing of the Fitbit, even if the employee does not reach the desired number of steps, nevertheless has well-being benefits is that the device itself may raise awareness of one's physical activity and the importance of a healthy lifestyle that includes physical movement. Particularly in a society (the US, in this case) often characterized as "coach potatoes", as immobile, and overweight, awareness of, and constant reminder of, the importance of physical activity might encourage the wearer to undertake more activity. Thus, even if the activity does not reach the targeted level, it might nevertheless represent a vast improvement and hence contribute to both well-being and health.

This finding has interesting implications for corporate wellness programs, especially with regards to employee participation. In our study, we asked 
non-participants at FIT Bank in a survey why they did not participate in the Fitbit aspect of the corporate wellness program. The majority of respondents indicated that they would like to participate, but are not able to due to an injury or a health condition that precludes them from walking and engaging in extended exercise. However, our results indicate that companies can impact employee well-being just by using the Fitbit features available. As such, corporate wellness programs could benefit by encouraging those who cannot achieve a high step count to wear the Fitbit and use other features such as tracking their sleep, connecting with other users, or tracking calories consumed. Finding ways to include employees who may be unable to participate in physical activity would help ensure that the entire workforce had an opportunity could improve their well-being and health. Furthermore, having a wellness program that has options for all employees, regardless of their health status or ability to do physical activity, would increase participation in a wellness program and, in the end, elevate the wellbeing and health of the workforce.

Although step count does have a positive impact on employee well-being and health, Fitbit use does have a positive impact on employee well-being and ultimately, their health, regardless of step count. Our study confirms other research which posits that exercise will improve health and that increasing the physical activity of employees should remain an important goal of corporate wellness programs. However, step count should not be the only focus. What matters is that individuals use wearable fitness devices and the features available to them. This means that corporate wellness programs should emphasize participation in wearable fitness initiatives, not just results. Engaging with wearable fitness trackers can connect users and provide support and motivation to participants as they strive for a healthy lifestyle.

\subsection{Limitations and future research}

We acknowledge that this research has limitations. The first is that our data sample is from one organization, which may limit the generalizability of our findings to other corporate wellness programs. The wellness program at FIT Bank has competitions that encourage employee participation and socialization. However, corporate wellness programs are very diverse. Some companies may not use wearable fitness devices, while others may not have any incentives to make health choices. Another limitation of our study is the R2 $=.136$ of extended use on step count. Given that this research is exploratory, we hope that future research will help uncover other predictors of physical activity, or step count. Another limitation is that some participants may have found ways to fake step count, which would artificially raise their physical activity tracked by the device. With Fitbits worn on the wrist, some activities, such as cooking or playing the piano, may be counted as a step when no step was taken.

Future research on wearable fitness trackers and corporate wellness programs might consider investigating the extent or depth of use of fitness devices and the impact on physical activity. Also, exploring which features have the highest impact on physical activity and well-being would be of interest for future studies. While we were able to look at current feature use, this may change over time. Longitudinal studies on wearable fitness devices suggest that a third of users stop using their devices within six months of purchasing the device [26]. In order to enjoy the benefits associated with Fitbit use, including health and well-being, corporate wellness program managers will have to find ways to encourage participants to keep wearing their devices to remain engaged in the program. Finally, another area of future research is to investigate the impact of Fitbit use on a corporate level. For example, how does wellness programs with wearable technology impact company insurance premiums and employee absenteeism? Also, research examining how companies are utilizing data from wearable fitness devices would also be useful.

\section{References}

[1] Agarwal, R., Guodong Gao, DesRoches, C., and Jha, A.K. The Digital Transformation of Healthcare: Current Status and the Road Ahead. Information Systems Research 21, 4 (2010), 796-809.

[2] Baptiste, N.R. Tightening the link between employee wellbeing at work and performance: A new dimension for HRM. Management Decision 46, 2 (2008), 284-309.

[3] Bort-Roig, J., Gilson, N.D., Puig-Ribera, A., Contreras, R.S., and Trost, S.G. Measuring and Influencing Physical Activity with Smartphone Technology: A Systematic Review. Sports Medicine 44, 5 (2014), 671-686.

[4] Bravata DM, Smith-Spangler C, Sundaram V, and et al. Using pedometers to increase physical activity and improve health: A systematic review. JAMA 298, 19 (2007), 2296-2304. 
[5] Butler, J. and Kern, M., L. The PERMA-Profiler: A brief multidimensional measure of flourishing. 2014. http://www.peggykern.org/questionnaires.html.

[6] Carter, M., Petter, S., and Retamales, J.A. Exploring the Relationship between IT Use and Wellbeing. (2015).

[7] Chin, W. The Partial Least Squares Approach to Structural Equation Modeling. In G.A. Marcoulides, ed., Modern Methods for Business Research. Psychology Press, Mahwah, N.J., 1998, 295-336.

[8] Chin, W.W., Marcolin, B.L., and Newsted, P.R. A Partial Least Squares Latent Variable Modeling Approach for Measuring Interaction Effects: Results from a Monte Carlo Simulation Study and an Electronic-Mail Emotion/Adoption Study. Information Systems Research 14, 2 (2003), 189-217.

[9] Choi, B.C.K., Pak, A.W.P., and Choi, J.C.L. Daily step goal of 10,000 steps: A literature review. Clinical \& Investigative Medicine 30, 3 (2007), 146151.

[10] Cohen, J. A power primer. Psychological Bulletin 112, 1 (1992), 155-159.

[11] Conn, V.S., Hafdahl, A.R., Cooper, P.S., Brown, L.M., and Lusk, S.L. Meta-Analysis of Workplace Physical Activity Interventions. American Journal of Preventive Medicine 37, 4 (2009), 330-339.

[12] Conroy, D.E., Yang, C.-H., and Maher, J.P. Behavior Change Techniques in Top-Ranked Mobile Apps for Physical Activity. American Journal of Preventive Medicine 46, 6 (2014), 649-652.

[13] Diener, E. and Chan, M.Y. Happy People Live Longer: Subjective Well-Being Contributes to Health and Longevity. Applied Psychology: Health and Well-Being 3, 1 (2011), 1-43.

[14] Direito, A., Jiang, Y., Whittaker, R., and Maddison, R. Smartphone apps to improve fitness and increase physical activity among young people: protocol of the Apps for IMproving FITness (AIMFIT) randomized controlled trial. BMC Public Health 15, (2015), 635.

[15] Etkin, J. The Hidden Cost of Personal Quantification. Journal of Consumer Research 42, 6 (2016), 967-984.
[16] Falkenberg, L.E. Employee Fitness Programs: Their Impact on the Employee and the Organization. Academy of Management Review 12, 3 (1987), 511522.

[17] Fitbit Inc. Fitbit for Corporate Wellness. Fitbit, Inc., San Francisco, CA, 2015.

[18] Fox, K.R. The influence of physical activity on mental well-being. Public Health Nutrition 2, Supplement 3a (1999), 411-418.

[19] Fox, S. and Duggan, M. Tracking for Health. Pew Research Center: Internet, Science \& Tech. http://www.pewinternet.org/2013/01/28/tracking-forhealth/.

[20] Ganju, K.K., Pavlou, P.A., and Banker, R.D. Does Information and Communication Technology Lead to the Well-Being of Nations? A Country-Level Empirical Investigation. MIS Quarterly 40, 2 (2016), 417-A10.

[21] Hair, J.F. Multivariate data analysis. Prentice Hall, Upper Saddle River, NJ, 2010.

[22] Hair, J.F., Hult, G.T.M., Ringle, C., and Sarstedt, M. A Primer on Partial Least Squares Structural Equation Modeling. SAGE Publications, Inc, Los Angeles, 2013.

[23] Hamper, A. A Context Aware Mobile Application for Physical Activity Promotion. IEEE (2015), 3197-3206.

[24] Hone, L.C., Jarden, A., Schofield, G., and Duncan, S. Measuring flourishing: The impact of operational definitions on the prevalence of high levels of wellbeing. International Journal of Wellbeing 4, 1 (2014), 62-90.

[25] Humphreys, B.R., McLeod, L., and Ruseski, J.E. Physical Activity and Health Outcomes: Evidence from Canada. Health Economics 23, 1 (2014), 33-54.

[26] Ledger, D. Inside Wearables-Part-2. EndeavourPartners, 2014.

[27] Levy, H. Wearable Technology Beyond Smartwatches. Smarter With Gartner, 2015. http://www.gartner.com/smarterwithgartner/wearable -technology-beyond-smartwatches-3/. 
[28] Lin, C.I.C., Feng-Yang Kuo, and Myers, M.D. Extending ICT4D Studies: The Value of Critical Research. MIS Quarterly 39, 3 (2015), 697-712.

[29] Lovett, N. and Lovett, T. Wellbeing in Education: Staff Matter. International Journal of Social Science and Humanity 6, 2 (2016), 107-112.

[30] Martin, J.A. Pros and Cons of Using Fitness Trackers for Employee Wellness. CIO, 2014. http://www.cio.com/article/2377723/it-strategy/prosand-cons-of-using-fitness-trackers-for-employeewellness.html.

[31] McCraty, R., Atkinson, M., Tiller, W.A., Rein, G., and Watkins, A.D. The effects of emotions on short-term power spectrum analysis of heart rate variability. The American Journal of Cardiology 76, 14 (1995), 1089-1093.

[32] Merrill, R.M., Aldana, S.G., Pope, J.E., et al. Self-Rated Job Performance and Absenteeism According to Employee Engagement, Health Behaviors, and Physical Health: Journal of Occupational and Environmental Medicine 55, 1 (2013), 10-18.

[33] Morris, M.G. and Venkatesh, V. Job Characteristics and Job Satisfaction: Understanding the Role of Enterprise Resource Planning System Implementation. MIS Quarterly 34, 1 (2010), 143161.

[34] Olson, P. Fitbit's Game Plan For Making Your Company Healthy. Forbes, 2016.

http://www.forbes.com/sites/parmyolson/2016/01/08/ fitbit-wearables-corporate-wellness/.

[35] Patel MS, Asch DA, and Volpp KG. WEarable devices as facilitators, not drivers, of health behavior change. JAMA 313, 5 (2015), 459-460.

[36] Penedo, F.J. and Dahn, J.R. Exercise and wellbeing: a review of mental and physical health benefits associated with physical activity. Current Opinion in Psychiatry 18, 2 (2005), 189-193.

[37] Saga, V.L. and Zmud, R.W. The Nature and Determinants of IT Acceptance, Routinization, and Infusion. Proceedings of the IFIP TC8 Working Conference on Diffusion, Transfer and Implementation of Information Technology, Elsevier Science Inc. (1994), 67-86.
[38] Seligman, M.E.P. Flourish: A Visionary New Understanding of Happiness and Well-being. Atria Books, New York, 2012.

[39] Soane, E., Shantz, A., Alfes, K., Truss, C., Rees, C., and Gatenby, M. The Association of Meaningfulness, Well-Being, and Engagement with Absenteeism: A Moderated Mediation Model.

Human Resource Management 52, 3 (2013), 441456.

[40] Solon, O. Wearable Technology Creeps Into The Workplace. Bloomberg.com, 2015. http://www.bloomberg.com/news/articles/2015-0807/wearable-technology-creeps-into-the-workplace.

[41] Springbuk. Targeted Engagement Study. Springbuk Report, 2015.

http://www.springbuk.com/targeted-engagementstudy/.

[42] Swan, M. The Quantified Self: Fundamental Disruption in Big Data Science and Biological Discovery. Big Data 1, 2 (2013), 85-99.

[43] Varo, J.J., Martínez-González, M.A., IralaEstévez, J. de, Kearney, J., Gibney, M., and Martínez, J.A. Distribution and determinants of sedentary lifestyles in the European Union. International Journal of Epidemiology 32, 1 (2003), 138-146.

[44] Williams, J. and MacKinnon, D.P. Resampling and Distribution of the Product Methods for Testing Indirect Effects in Complex Models. Structural Equation Modeling: A Multidisciplinary Journal 15, 1 (2008), 23-51.

[45] Wright, T.A. To be or not to be [happy]: The role of employee well-being. The Academy of Management Perspectives 20, 3 (2006), 118-120.

[46] Counting every moment. The Economist 402, 2012, 22(US).

[47] CDC - Workplace Health Toolkit Model. http://www.cdc.gov/workplacehealthpromotion/mode 1/index.html. 